\title{
Configuración de la región sanitaria de la zona Altos TSOTSIL-TSELTAL: PANORAMA ACTUAL DE LA BÚSQUEDA DE SALUD
}

\author{
Configuration of the Health Region of the Altos \\ Tsotsil-Tseltal Area: Current Panorama of the Search \\ FOR HEALTH
}

\author{
Rocío Marisela Ruíz Ruíz* \\ José Enrique Eroza Solana** \\ Jorge Magaña Ochoa**** \\ doi: https://doi.org/10.31644/ED.V9.N1.2022.A10
}

\begin{abstract}
Resumen: En la construcción de una región participan diversos actores sociales e institucionales, que en el devenir histórico construyen y reconstruyen procesos de forma individual y colectiva. En la zona denominada Altos Tsotsil-Tseltal se ha configurado una región sanitaria, constituida por procesos salubristas relacionales entre quienes brindan atención a la salud y quienes emprenden la búsqueda de la misma. En este artículo se analizan los procesos históricos que han conformado la oferta de opciones de atención a la salud y las formas en que la población las articula, mediante sus trayectorias de atención, lo que confiere a esta zona una dinámica sociocultural propia al configurar una región sanitaria relativamente distintiva.
\end{abstract}

Palabras clave: región, procesos sanitarios, atención a la salud, zona Altos Tsotsil-Tseltal.

Abstract: Various social and institutional actors participate in the construction of a region, which through historical processes has been individually and collectively shaped and reshaped. In the area known as theTsotsil-Tseltal Highlands, a health system-related region has been fashioned, constituted by relational processes between those who provide health care and those who undertake the search of health. This paper, deals with the historical processes that have configured the offer

* Doctorante en Estudios Regionales, Universidad Autónoma de Chiapas, México. ORCiD: https://orcid.org/00000001-5968-9402 Correo-e: rocio.ruiz1806@gmail.com.

* Dr. en Filosofía, profesor-investigador Centro de Investigaciones y Estudios Superiores en Antropología Social Región Sureste, México. ORCiD: https://orcid.org/0000-0002-1430-6022 Correo-e: enriquesol@yahoo.com.

* Dr. en Antropología Social y Cultural, profesor-investigador Facultad de Ciencias Sociales, Universidad Autónoma de Chiapas, México. ORCiD: https://orcid.org/0000-0002-9424-2814 Correo-e: jorge.magana@unach.mx.

Fecha de recepción: 27/05/2021. Fecha de aceptación: 22/12/2021. Fecha de publicación: 31/01/2022.

(cc) BY-NC-ND

EntreDiversidades. Revista de Ciencias Sociales y Humanidades, Vol. 9, Núm. 1 (18), enero-junio 2022. Páginas: $247-271$ ISSN-e: 2007-7610. https://doi.org/10.31644/ED.V9.N1.2022.A10 
of different health care options and the articulation that the population makes of them, through their health seeking trajectories, which confers to this zone its own socio-cultural dynamic by forming a relatively distinctive health system region.

Keywords: region, health processes, health care, Altos Tsotsil-Tseltal zone.

\section{Introducción}

Se analiza el transitar histórico y relacional del papel de individuos, organizaciones e instituciones, entre prácticas de quiénes han brindado y brindan atención a la salud y de aquellos a quienes han sido y son dirigidas, para construir la región sanitaria de los Altos de Chiapas. En primer término, a partir de información de fuentes documentales y de experiencias de actores sociales que han sido y son participes de organizaciones e instituciones de atención a la salud. Posteriormente, revisamos el panorama actual de búsqueda de salud en la zona, mediante información registrada en campo de dos estudios. ${ }^{1}$

Iniciamos planteando el concepto de región, a partir del que se formula el de región sanitaria, para enseguida emprender un recorrido histórico a lo largo de la implementación de políticas públicas en salud, de iniciativas por parte de organizaciones indígenas y civiles, de instituciones de salud públicas y privadas, de prácticas tradicionales y religiosas referidas a la salud, de medicinas alternativas y complementarias, de empresas productoras y comercializadoras de multivitamínicos, grupos de autoayuda y prácticas de autocuidado. Por último, documentamos y reflexionamos en torno a la articulación de opciones de atención a la salud que, como efecto de procesos socioculturales relativamente recientes, las personas emprenden en el cometido de resolver sus problemáticas en salud en la región.

Los testimonios en los que se basa el análisis de la articulación de opciones de atención a la salud se refieren a datos relativos a trayectorias de atención registradas mediante dos investigaciones, una realizada entre 2008 y 2016, denominada "Procesos de búsqueda de atención entre Tsotsiles y Tseltales de los Altos de Chiapas", ${ }^{2}$ y otra realizada durante 2020 y 2021 con personas que recibieron atención en el Hospital de las Culturas ${ }^{3}$ y que refirieron haber buscado otras opciones para atender sus problemas de salud. ${ }^{4}$

La metodología para recabar información sobre actores institucionales, así como vinculada a programas de salud y a organizaciones, se centró en entrevistas semiestructuradas. Los entrevistados fueron contactados mediante la estrategia de bola de nieve. Se entrevistó a personal que laboraba

\footnotetext{
${ }^{1}$ En dos diferentes periodos, uno de 2008 a 2016 y otro de 2020 a 2021.

${ }^{2}$ Realizada por uno de los autores, el Dr. Enrique Eroza, docente investigador del Centro de Investigaciones y Estudios Superiores en Antropología Social, Unidad Regional Sureste (CIESAS Sureste).

${ }^{3}$ Hospital general de segundo nivel de atención, que se encuentra ubicado en la ciudad de San Cristóbal de Las Casas, Chiapas, y que brinda cobertura de atención a la zona Altos Tsotsil-Tseltal, principalmente.

${ }^{4}$ Realizada por una de las autoras del presente artículo, la Mtra. Rocío Ruíz, doctorante de Estudios Regionales de la Universidad Autónoma de Chiapas (UNACH), como parte de su proyecto de tesis "De encuentros ficticios a desencuentros reales: saberes y significados de pacientes sobre su padecimiento/enfermedad en el Sistema hospitalario del Hospital de Las Culturas".
}

EntreDiversidades. Revista de Ciencias Sociales y Humanidades, Vol. 9, Núm. 1 (18), enero-junio 2022. Páginas: 247-271 ISSN-e: 2007-7610. https://doi.org/10.31644/ED.V9.N1.2022.A10 
en el Distrito de Salud no. II, ${ }^{5}$ en el Instituto Nacional de los Pueblos Indígenas (INPI), ${ }^{6}$ de algunas Organizaciones civiles y religiosas y a personal de salud del Hospital de Las Culturas. Testimonios de las trayectorias del padecimiento se obtuvieron con entrevistas a profundidad y observación directa.

\section{Región sanitaria, una construcción histórica}

Dentro de un territorio las interrelaciones se gestan de manera conjunta en los ámbitos históricos, culturales, sociales, económicos y políticos, y configuran una región específica en la que interactúan procesos estructurales y socioculturales en diferentes escalas. Las regiones constituidas desde una visión político administrativa, como lo es la zona V Altos Tsotsil-Tseltal, ${ }^{7}$ otorgan un referente geográfico, político y económico, así como cultural, pero, su división no explicita los procesos que históricamente se fueron gestando a escalas locales, regionales, nacionales y globales por parte de los propios actores sociales.

Se alude así al concepto de región como una construcción resultante de procesos individuales y colectivos, como espacio vivido por y desde los sujetos dentro de un mismo espacio territorial (García, 2006). Dentro de la región existe una cultura con contenido social y político-económico que se produce mediante interacciones humanas (Lomnitz, 1995), se construye históricamente y se crea una estructura con procesos distintivos (Albet, 1993).

Partiendo de esta premisa, el presente texto analiza la construcción de una región sanitaria, en términos de los procesos salubristas dirigidos a lo largo del tiempo a la población usuaria, inicialmente a los indígenas y ulteriormente también a la no indígena. Máxime los que tuvieron y tienen lugar en San Cristóbal de Las Casas, cabecera regional de Los Altos de Chiapas, donde confluye una amplia variedad de opciones de atención a la salud ${ }^{8}$ y no solamente la biomédica. En esta ciudad se concentran servicios asistenciales, sociales y comerciales, y concurren personas de toda la región.

Es menester aclarar que la biomedicina es entendida como el sistema médico hegemónico que enfatiza el modelo biológico y el control tecnológico de la enfermedad como entidad, pero en detrimento del análisis de las correlaciones y/o determinantes mentales, sociales y culturales de la enfermedad. Es una medicina científica y cosmopolita, porque es académica, se trasmite a través de la educación universitaria, y, debido a su propia hegemonía, se apropia de los recursos

\footnotetext{
${ }^{5}$ Denominado hasta el año 2019 Jurisdicción Sanitaria no. II.

${ }^{6}$ Antes de 2003 se denominaba Instituto Nacional Indigenista (INI), posterior a este año Comisión Nacional para el Desarrollo de los Pueblos Indígenas y, actualmente, INPI.

${ }^{7}$ Una de las 15 zonas político-administrativas del estado de Chiapas, compuesta por 17 municipios localizados en la parte central del estado, y cuya cabecera regional es la ciudad de San Cristóbal de Las Casas, Chiapas. Aunque la regionalización administrativa ha variado, a partir de mayo de 2011 se denominó así de acuerdo a la última reforma (Ruíz, 2018).

${ }^{8}$ Información basada en el proyecto de tesis "De encuentros ficticios a desencuentros reales: saberes y significados de pacientes sobre su padecimiento/enfermedad en el Sistema hospitalario del Hospital de Las Culturas” (Ruíz, 2021), para dar cuenta de las otras opciones de atención a la salud, además de la biomedicina, que se otorga en el Hospital de Las Culturas, y que los pacientes hacen uso antes o después de asistir al servicio hospitalario.
}

EntreDiversidades. Revista de Ciencias Sociales y Humanidades, Vol. 9, Núm. 1 (18), enero-junio 2022. Páginas: $247-271$ ISSN-e: 2007-7610. https://doi.org/10.31644/ED.V9.N1.2022.A10 
de otras prácticas médicas, las fusiona y las hace parte de la suya. Además, no solo se centra en la conceptualización orgánica de la enfermedad, también tiene su propia forma de tratarla, a través de medicamentos e intervenciones físicas para aminorar los síntomas, es decir, con alopatía; y a partir de la bioquímica se aprovecha de la farmacopea a nivel de industria para generar procesos mercantiles desde una lógica capitalista. En el presente artículo se hace referencia a la biomedicina como sinónimo de medicina científica, cosmopolita y medicina alópata (Menéndez, 2016; Eroza y Carrasco, 2019; Magaña y Rojas, 2020).

Por último, en los procesos sanitarios que se documentan y analizan han participado y participan diversos actores y usuarios, quienes a lo largo del tiempo han interactuado en la conformación relativamente particular de la región sanitaria.

Si bien partimos del análisis histórico de las problemáticas de salud de la población indígena y de las políticas institucionales en materia de salud, también documentamos y revisamos la riqueza y complejidad que hoy día expresa la población de los Altos de Chiapas en lo concerniente a las prácticas de atención a su salud, tanto la población indígena como no indígena.

\section{Políticas sanitarias y organizaciones indígenas}

En la década de los treinta del siglo XX se suscitó en México un movimiento médico tendiente a erradicar a los curanderos urbanos e indígenas; junto a estas acciones, el gobierno cardenista retomó la estrategia integracionista de Manuel Gamio de 1915, quien la proponía como una solución al problema indígena en México. En 1935 se crearon las Agencias del Departamento de Acción Social y Cultural y de Protección al Indígena abriéndose una en San Cristóbal de Las Casas. No obstante, a fines de los años cuarenta, dicho programa desapareció y se implantó una política indigenista integracionista dependiente a nivel federal (Kohler, 1975; Villa Rojas, 1976; Campos, 1996).

En 1940, en Pátzcuaro, Michoacán, se celebró el Primer Congreso Interamericano Indigenista. En este se establecieron las bases para el desarrollo de la política indigenista continental. A raíz de dicho evento, el gobierno mexicano inauguró en 1948 el Instituto Nacional Indigenista (INI) y su primer centro coordinador en San Cristóbal de Las Casas (Page, 2002).

También arribaron a la región iglesias evangélicas y protestantes. En 1944 misioneras presbiterianas, amén de evangelizar, prestaban servicios de salud a la población en localidades de Oxchuc (Harman, 1969). Hacia los setenta, las misioneras contaban con clínicas equipadas y en los ochenta fundaron una escuela de capacitación en salud (Villa Rojas, 1990).

En 1957 la Iglesia católica también comenzó a emprender acciones asistenciales en salud, introduciendo nociones de higiene (Harman, 1969). En el año 1988 constituyó el área diocesana de salud con agentes de pastoral encargados de planear, coordinar y capacitar a agentes de salud para control de la natalidad con métodos naturales, salud comunitaria, manejo de enfermedades frecuentes, rescate del sistema tradicional y el manejo de un botiquín básico (Freyermuth, 1993).

En 1974 la voz de las comunidades indígenas se había escuchado en el Primer Congreso Indígena de Chiapas, “Tierra, comercio, educación y salud en San Cristóbal de Las Casas,

EntreDiversidades. Revista de Ciencias Sociales y Humanidades, Vol. 9, Núm. 1 (18), enero-junio 2022. Páginas: $247-271$ ISSN-e: 2007-7610. https://doi.org/10.31644/ED.V9.N1.2022.A10 
Chiapas", en el que el representante de la Delegación Tsotsil planteó algunas demandas de su población en relación a sus condiciones de salud (Lozoya, Velázquez y Flores, 1988). ${ }^{9}$

Ante la influencia de agencias internacionales respecto a la atención en salud para toda la población y con la lógica de un modelo económico neoliberal, el Estado mexicano instauró políticas sanitarias que incluían la medicina tradicional para la atención primaria a la salud. ${ }^{10}$ Desde entonces se iniciaría una serie de políticas del Sector Salud que, aún con diversas designaciones, según el periodo político, han sido reiterativas en contenido y, a la larga, fallidas.

Durante los años setenta, también se implementaron programas a nivel federal que tuvieron protagonismo en Chiapas; en 1977 se creó la Coordinación General del Plan Nacional de Zonas Deprimidas y Grupos Marginados (COPLAMAR), en 1979 suscribió un convenio con el Instituto Mexicano del Seguro Social (IMSS) y se convirtió en el Programa Nacional de Solidaridad Social por Cooperación Comunitaria (IMSS-COPLAMAR), y en 1982 se emprendió un proyecto denominado "Programa de interrelación de la medicina tradicional con el Programa Nacional de Solidaridad Social por Cooperación Comunitaria IMSS-COPLAMAR", con la finalidad de proporcionar herramientas tendientes a facilitar el acercamiento de la medicina indígena con la alópata (Viesca, 1987; Page, 2002), ${ }^{11}$ aunque la segunda prevaleció al final.

En 1979 el Centro Coordinador Tzeltal-Tzotzil de San Cristóbal echó a andar el proyecto "Medicinas Paralelas" — que a la postre se extendería a otros centros coordinadores_, a partir de entonces se comenzaron a realizar reuniones con médicos tradicionales y parteras en los que se pretendía el intercambio de experiencias entre médicos indígenas de distintas regiones. ${ }^{12}$ Pero hacia 1982 el INI ya no contaba con presupuesto y dio por terminado el proyecto, que pasó a ser parte de la Secretaría de Salud (SSA) (Page, 2002). En 1985 el convenio con la SSA finalizó y los médicos indígenas se retiraron del programa (Freyermuth, 1993; Page, 2002).

Estos acontecimientos llevaron a los médicos tsotsiles y tseltales y a los promotores de salud, también miembros de dichas etnias, a crear en 1985 la Organización de Médicos Indígenas del

\footnotetext{
${ }^{9}$ Expresó que los centros de salud estaban concentrados en la ciudad, que existía falta de comunicación con los médicos, sufrían de mal trato del personal de enfermería, que no se tomaban en cuenta sus medicinas, lidiaban con costos elevados, abusos y discriminación, pidió que se enseñara la medicina preventiva, preparación de enfermeros indígenas, que se estudiara el modo de integrar ambas medicinas y que aprendiera el personal de salud institucional las lenguas indígenas (Lozoya, Velázquez y Flores, 1988).

${ }^{10}$ La Organización Mundial de la Salud (OMS) buscó engarzar sus propuestas con los programas de salud, apoyándose de las recomendaciones que se dieron en la Declaración de Alma-Ata sobre "Atención Primaria en Salud" en 1978, y en la estrategia global de "Salud para todos en el año 2000"; la OMS se centró en extender los servicios de salud y en la atención primaria de Salud tomando los recursos locales como la medicina tradicional, con inclusión de médicos, enfermeras, parteras, auxiliares y trabajadores de la comunidad, siempre y cuando recibieran adiestramiento social y técnico para trabajar con un equipo de salud. México firma dicha declaración, y el Estado lo toma como una oportunidad para pasar la responsabilidad de la atención primaria a la población y ahorrar recursos (Lozoya, Velázquez y Flores, 1988; Freyermuth, 1993; Page, 2002).

${ }^{11}$ Esta fue la primera vez que se oficializó la inclusión de la medicina tradicional dentro del Sistema Nacional de Salud (Page, 2002).

${ }^{12}$ Entrevista a Rafael Alarcón Lavín en septiembre de 2020. Médico en el INI, fue coordinador del Centro de Ocosingo, y, posteriormente, asesor de la Organización de Médicos Indígenas del Estado de Chiapas, A. C. (OMIECH) desde 1986.
}

EntreDiversidades. Revista de Ciencias Sociales y Humanidades, Vol. 9, Núm. 1 (18), enero-junio 2022. Páginas: $247-271$ ISSN-e: 2007-7610. https://doi.org/10.31644/ED.V9.N1.2022.A10 
Estado de Chiapas, A. C. (OMIECH) ${ }^{13}$ (Page, 2002). Comenzó a tener asesores del INI que representaban el vínculo con la biomedicina, pero cuyo rol era mantener una visión amplia del contexto sanitario cultural de ambas medicinas. Seguidamente, crearon el "Museo de la Medicina Maya”, donde aún proporcionan atención los médicos indígenas tsotsiles y tseltales. ${ }^{14}$

Ulteriormente, se creó la Organización de Terapeutas Indígenas Tzeltales (ODETIT) en Oxchuc, a fin de preservar y fortalecer la medicina indígena tseltal, aunque tuvieron problemas para recibir asesorías y tramitación de proyectos (Freyermuth, 1993; Page, 2002).

En 1991 conformaron una red nacional de organizaciones de médicos indígenas y un órgano coordinador de las organizaciones, el Consejo Nacional de Médicos Indígenas Tradicionales (CONAMIT).${ }^{15} \mathrm{~A}$ través de la OMIECH y el CONAMIT se hicieron convenios con el sector salud, que tampoco lograron articular la medicina indígena y la alópata (Page, 2002).

El camino que siguió después la OMIECH fue un tanto infructuoso, en 2003 dejaron de recibir recursos. Los directivos del INI también cambiaron la línea de apoyo a la medicina indígena y comenzaron a trabajar en coordinación con el Sector Salud para tener más recursos. ${ }^{16}$ La organización se mantiene vigente, pero con dificultades debido al camino por el que ha transitado ante un sector político-económico-sanitario que sigue sin reconocer ni entender los saberes y las prácticas en salud de la población indígena. Quienes acuñaron el término de medicina tradicional, lo hicieron mediante una visión reduccionista, pues en realidad las prácticas indígenas al respecto participan de la totalidad de la vida social de las comunidades y más allá de ellas, por lo mismo, sus prácticas en salud no pueden considerarse vis a vis con la medicina científica; tampoco, por la misma razón, han tenido alguna vez cabida en un mismo espacio, al menos en términos de práctica colaborativa y complementaria (Knipper, 2006).

Es de mencionar, también, que, por su parte, las organizaciones de médicos tradicionales han aprendido a jugar el juego planteado por las instituciones, para beneficiarse del mismo, por lo que llegan a utilizar ciertos discursos de lo cultural, terminando, a fin de cuentas, por alimentar un imaginario que nos presenta una cara esencialista de los grupos étnicos y de su cultura.

\section{Organizaciones de la Sociedad Civil}

Las décadas de los ochenta y noventa del siglo XX estuvieron marcadas por movimientos sociales y cambios propuestos desde la sociedad civil, así como por el nacimiento de organizaciones no gubernamentales (ONG), como el Colectivo de Mujeres (COLEM) de San Cristóbal de Las Casas, que se fundó en 1989 para la defensa de los derechos humanos y de la violencia contra las mujeres, sobre todo indígenas, y proporcionaba atención médica y programas de capacitación con una perspectiva intercultural (SIPAZ, 2014), y la organización Asesoría, Capacitación y Asistencia en Salud, A.C. (ACASAC), que brinda atención en materia de salud a las comunidades

\footnotetext{
${ }^{13}$ Rafael Alarcón Lavín, comunicación personal en septiembre de 2020.

${ }^{14}$ Rafael Alarcón Lavín, comunicación personal, en septiembre de 2020.

${ }^{15}$ Que llegó a agrupar a 57 organizaciones de médicos indígenas del país.

${ }^{16}$ Rafael Alarcón Lavín, comunicación personal, en septiembre de 2020.
}

EntreDiversidades. Revista de Ciencias Sociales y Humanidades, Vol. 9, Núm. 1 (18), enero-junio 2022. Páginas: $247-271$ ISSN-e: 2007-7610. https://doi.org/10.31644/ED.V9.N1.2022.A10 
y atención a mujeres e infantes víctimas de violencia — cuenta con el Centro de Atención contra la Violencia Familiar (CAVIF) ${ }^{17}$ y con un Refugio para las Mujeres víctimas de violencia, básicamente indígenas-.

Con el levantamiento del Ejercito Zapatista de Liberación Nacional (EZLN) emergieron otras Organizaciones, ligadas a la iglesia, con proyectos para la prevención, atención y promoción de la Salud. La Fundación León XIII fue una de ellas, fundada en 1994 por un grupo de personas ligadas al Instituto Mexicano de Doctrina Social Cristiana (IMDOSOC). Comenzó a atender necesidades de las comunidades indígenas de las zonas Altos y Selva, mediante la promoción de la salud con herbolaria, así como la elaboración y venta de artesanías. En 2012 trasformó su modelo quedando como base el programa de "Grupos de Ahorro". El de salud continuó, mas por falta de recursos dejaron de dar consultas en las comunidades. ${ }^{18}$

En 1998 una fundación de Monterrey estableció la Clínica Esquipulas, inicialmente ligada al obispo Samuel Ruiz, dada la necesidad que les planteó sobre salud. Hoy día proporciona consulta externa con servicios de medicina general, odontología general, odontopediatría, endodoncia, traumatología, reumatología, otorrinolaringología, ginecología, oftalmología, endocrinología, pediatría, psicología, laboratorio de análisis clínicos y rayos X. Todos los servicios se otorgan con cuotas de recuperación mínimas. Trabajan también con promotores de salud, y a través de ellos conocen las prácticas de los médicos tradicionales y parteras. ${ }^{19}$

La Fundación León XIII se alió posteriormente a una asociación civil denominada Sanando Heridas, creada en 2008. Otorga consultas médicas y medicamentos con una cuota de recuperación mínima en su propia sede. ${ }^{20}$ Trabajan en las localidades de Chenalhó, Zinacantán, Chamula, Teopisca y San Cristóbal de Las Casas. ${ }^{21}$ A partir de la pandemia de la covid-19 comenzaron a dar atención en San Cristóbal los días lunes y miércoles a cualquier persona. ${ }^{22}$

Estas organizaciones no son las únicas en la zona, ${ }^{23}$ existen varias, pero en ocasiones se han encontrado en competencia o duplicando acciones en las mismas localidades. ${ }^{24}$ Por esta razón, se creó en 2012 la Alianza por la salud, alimentación y nutrición (ASAN) en San Cristóbal, que

\footnotetext{
${ }^{17}$ Brinda los servicios de acompañamiento psicológico, jurídico y de trabajo social, realizan talleres, capacitaciones, pláticas y cursos y canalización a espacios de protección (CAVIF, 2021).

${ }^{18}$ Se obtuvo la información de la Fundación León XIII y sus acciones desde su inicio hasta la época actual, en una entrevista con la Coordinadora de San Cristóbal de Las Casas, el 3 de febrero de 2021.

${ }^{19}$ Información obtenida en entrevista al director de la Clínica Esquipulas, en enero de 2021.

${ }^{20}$ En la actualidad existen tres Centros de la Fundación León XIII, en Chiapas en San Cristóbal de Las Casas, en Oaxaca en Pinotepa Nacional y en Guerrero en Santa María Huatulco.

${ }^{21} \mathrm{La}$ coordinadora refiere que originalmente ya tenían relaciones de confianza con algunas localidades, ya que conocían gente de cooperativas u organizaciones indígenas, y establecieron acuerdos de trabajo con las autoridades.

${ }^{22}$ Información obtenida con la Coordinadora de Sanando Heridas, en enero de 2021.

${ }^{23}$ Por limitaciones de presupuesto, tiempo y la pandemia de la covid-19 no se realizaron entrevistas a otras organizaciones.

${ }^{24}$ De acuerdo a la coordinadora de la Fundación León XIII.
}

EntreDiversidades. Revista de Ciencias Sociales y Humanidades, Vol. 9, Núm. 1 (18), enero-junio 2022. Páginas: $247-271$ ISSN-e: 2007-7610. https://doi.org/10.31644/ED.V9.N1.2022.A10 
Rocío Marisela Ruiz Ruiz

José Enrique Eroza Solana

Jorge Magaña Ochoa
Configuración de la región sanitaria de la zona Altos Tsotsil-Tseltal: panorama...

trabaja como un actor colectivo de 19 organizaciones ${ }^{25}$ mediante tres ejes temáticos: agua, salud y nutrición, así como seguridad alimentaria, en distintas áreas de influencia del estado.

Otras ONG brindan apoyo a mujeres embarazadas en situación de violencia y calle, para la atención del parto con parteras tradicionales y el puerperio, como el Hogar Comunitario Yach'il Antzetic. ${ }^{26}$ Dan servicio gratuito a cambio de colaboración en tareas de limpieza en casa, por parte de las mujeres (Morales, 2021).

Respecto a estas organizaciones, se advierte que los procesos sanitarios están entrelazados con dinámicas socio-políticas. El movimiento zapatista dio pie a la intervención de sectores no gubernamentales, aún a nivel internacional; si bien, con una óptica centrada en problemáticas sociales y tentativas tendientes a promover la relación con saberes y prácticas locales basadas en el diálogo con las comunidades, sus perspectivas y acciones, no han dejado de ser una expresión de la cultura biomédica:

En el tiempo que hubo consulta médica aquí estaba el médico herbolario, [...] hay algunas comunidades que tienen muy arraigada la medicina tradicional, pensando en eso es que la fundación cuando daba consultas tenía aquí al médico tradicional, había un consultorio de medicina herbolaria, incluso teníamos un huerto de plantas medicinales, y también era huesero [...] hay personas específicas que dicen: "prefiero que sea medicamento natural”, pero también la parte de cómo convences a esa gente que además de la medicina tradicional, necesita la medicina alópata (Entrevista a la coordinadora de la Fundación León XIII, enero 2021).

No estamos peleados con la utilización de la medicina alópata junto con herbolaria, siempre y cuando no haya inconvenientes, o algunos otros tratamientos que por creencia puedan llevar [...] algunas cosas hemos aprendido entonces también ¿por qué no echar mano otras tantas? Tenemos que analizar y también somos críticos, y podemos decir aquí no; igual con la partería estamos de acuerdo, hasta hemos impulsado con el Tecnológico de Monterrey con la creación de casas de partería y mientras esté todo bien con la experiencia que ellas tienen, pues adelante (Entrevista al director de la Clínica Esquipulas, enero 2021).

\footnotetext{
${ }^{25}$ Pro Mazahua; Asociación Mexicana de Transformación Rural y Urbana, A. C. (AMEXTRA); Audes Milenio UNACH (de la Universidad Autónoma de Chiapas); Batsil Antsetik, A. C.; Fundación Cántaro Azul, A. C.; Centro Agropecuario para el Desarrollo Rural, A. C. (CEADER); Consultorías Integrales para el Desarrollo Rural Sustentable, S. C. (CONDINER); Diseño y Capacitación para el Desarrollo y las Microfinanzas S. C. (DICADEM); Foro para el Desarrollo Sustentable, A. C. (FORO); Fundación León XIII, I. A. P.; Alianza Pediátrica Global, Instituto de Liderazgo Simone de Beauvoir, A. C., Proyectos de Reciclaje y Acciones por la Naturaleza "PRANA", S. A. P. I. de C. V. (GPA); Sanando Heridas, A. C.; Salud y Nutrición, A. C. (SANUT); Save the Children, Chiapas, México; Facultad de Ciencias de la Nutrición y Alimentos, UNICACH (de la Universidad de Ciencias y Artes de Chiapas); y, por último, YAXALUM, Chiapas A. C. (ASAN, 2020).

${ }^{26}$ Fue fundado en 1996, en San Cristóbal de Las Casas, Chiapas, por María de la Luz Ruíz García para acompañar a mujeres indígenas y mestizas que viven un embarazo no planeado y violencia de género (Hogar Comunitario Yach’il Antzetic, 2021).
}

EntreDiversidades. Revista de Ciencias Sociales y Humanidades, Vol. 9, Núm. 1 (18), enero-junio 2022. Páginas: 247-271 ISSN-e: 2007-7610. https://doi.org/10.31644/ED.V9.N1.2022.A10 
Igual con los diferentes sistemas médicos que existen, porque los alópatas pensamos que solo la alopatía es medicina, entonces pues igual si la gente quiere probar la hierba, o quiere ir al Ilo' $l$ [curandero, en tsotsil] pues se respeta, se platica, se hace un diálogo: "mira, sí me parece", o "ve, pero tómate también esta medicina" (Entrevista a la coordinadora de Sanando Heridas, diciembre 2020).

\section{Espacios representados por el Sector Salud}

Otros espacios que forman parte de la región sanitaria en la zona Altos Tsotsil-Tseltal son los del Sector Salud, correspondientes al primer nivel de atención, segundo y tercer nivel. ${ }^{27}$

Por parte de la Secretaría de Salud (SSA) existen en la zona Altos, en diferentes municipios, unidades médicas exclusivamente de primer y segundo nivel de atención. ${ }^{28}$

Los hospitales, que corresponden al segundo nivel, son: 5 Hospitales Integrales Comunitarios, que se encuentran en Chamula, San Andrés Larráinzar, Oxchuc, Teopisca y Chalchihuitán, y dos Hospitales Generales, que se encuentran en San Cristóbal de Las Casas, el Hospital de Las Culturas y el Hospital de la Mujer. Estos últimos reciben pacientes de todos los demás municipios, y los refieren, cuando es necesaria atención subespecializada, a otros hospitales de segundo y tercer nivel en Tuxtla Gutiérrez y Tapachula; ${ }^{29}$ tal disyuntiva genera dificultades para la oportuna atención, ya que los gastos de traslado deben subsidiarlos los propios pacientes, quienes terminan por no hacerlo dado su falta de recursos. ${ }^{30}$

En el estudio realizado en dicho Hospital, encontramos que existen dificultades por falta de insumos, por el tipo de políticas institucionales y por un sistema burocrático vertical que depende de la SSA estatal y federal y de políticas internacionales. ${ }^{31}$

Otras instituciones del sistema de salud de la región sanitaria son el Instituto Mexicano del Seguro Social (IMSS), que atiende a trabajadores asalariados, patrones, y funciona con cuotas

\footnotetext{
${ }^{27}$ Los componentes del sistema de salud chiapaneco se encuentran, dada su organización, segmentados horizontalmente e integrados verticalmente en orden jerárquico. La estructura vertical se conforma, básicamente, por tres niveles de atención: el primer nivel opera como mínimo con un médico general, enfermería y técnico en salud; el segundo atiende situaciones de mayor complejidad; incluye uno o más hospitales con al menos cuatro especialidades básicas; y, por último,el tercer nivel se conforma por hospitales e institutos de alta especialidad (Jiménez y Núnez, 2016).

${ }^{28}$ De primer nivel: 2 Brigadas móviles, 40 Unidades móviles, 72 Centros de salud rurales básicos, 9 Centros de salud Urbanos básicos, 36 Casas de Salud, 6 Centros de Salud con servicios ampliados, 3 Centros de Salud con hospitalización, 3 Unidades de Especialidades Médicas (UNEMES), 2 Clínicas de especialidades. De segundo nivel: 1 Clínica de atención respiratoria (covid-19), 5 Hospitales Integrales Comunitarios y 2 Hospitales Generales (SSA, 2021).

${ }^{29}$ Información obtenida en entrevista a una Trabajadora Social del Hospital de Las Culturas, en enero de 2021.

${ }^{30}$ Información obtenida en entrevista a una Trabajadora social del Hospital de Las Culturas, en enero de 2021, y a dos pacientes del mismo Hospital en diciembre de 2020 y enero de 2021.

${ }^{31} \mathrm{~A}$ través de diversas entrevistas al personal de salud, administrativo y de vigilancia y limpieza, de diciembre de 2020 a febrero de 2021.
}

EntreDiversidades. Revista de Ciencias Sociales y Humanidades, Vol. 9, Núm. 1 (18), enero-junio 2022. Páginas: $247-271$ ISSN-e: 2007-7610. https://doi.org/10.31644/ED.V9.N1.2022.A10 
patronales, trabajadores y recursos del gobierno; ${ }^{32}$ el Instituto de Seguridad y Servicios Sociales de los Trabajadores del Estado (ISSSTE), que asiste a los empleados de las dependencias estatales; y el Instituto de Seguridad Social para los Trabajadores del Estado de Chiapas (ISSSTECH), que atiende al personal y sus familias de las dependencias del gobierno chiapaneco y la Secretaría de la Defensa Nacional (SEDENA). Tanto el IMSS, como el ISSSTE y el ISSSTECH operan con muchas limitantes en cuanto a recursos y atención oportuna.

La población no derechohabiente de las instituciones de seguridad social podía afiliarse al programa de protección social del Seguro Popular, fundado en 2003, pero en 2020 se sustituyó por el INSABI (Instituto de Salud para el Bienestar), que atiende a población abierta, por lo que se espera que en los próximos años la atención pública amplíe su cobertura a toda la población sin requerir afiliación.

\section{Política de interculturalidad en salud en el Hospital de Las Culturas}

Desde la creación de la Dirección de Interculturalidad en $2003,{ }^{33}$ se determinó que en la zona Altos Tsotsil-Tseltal las prácticas de atención deben regirse por lineamientos de interculturalidad. ${ }^{34}$ Sin embargo, estos se basan en criterios que se limitan a ciertos aspectos de orden cultural, cuyo enfoque no repercute en un mutuo entendimiento, ni, por tanto, enriquece la interacción entre los profesionales de la salud y las perspectivas y prácticas en salud de la población usuaria. ${ }^{35} \mathrm{~A}$ partir de esta política, la interculturalidad es un enfoque que la SSA le exige implementar al Hospital de Las Culturas, con el objetivo de ofrecer atención a la salud con pertinencia cultural no solo a la población indígena, sino en general (Ruíz, 2021).

\footnotetext{
${ }^{32}$ Tiene la sección comunitaria, conocida ahora como Bienestar, antes fue Prospera, Oportunidades, Progresa y COPLAMAR.

${ }^{33}$ Desde la SSA (2008) se plantea que la interculturalidad en la salud es una herramienta para comprender diferentes elementos asociados a la cultura de los usuarios, de las diversas prácticas terapéuticas y sistemas de atención a la salud a los que acude la población, como la medicina indígena, la acupuntura y la homeopatía. Los planteamientos se centran en cuatro estrategias para alcanzar la competencia cultural: capacitación/sensibilización del personal de salud, impulso y fortalecimiento de la medicina tradicional indígena, impulso y desarrollo de las medicinas complementarias y atención a la salud de los pueblos indígenas.

${ }^{34} \mathrm{El}$ concepto de interculturalidad emana de los movimientos sociales latinoamericanos en 1970, en una búsqueda por legitimar, defender y empoderar a los pueblos indígenas y cuestionar a los sectores que los excluían o discriminaban, pero se dejó de lado la transformación de las estructuras colonialistas con carácter monocultural y hegemónico (Eroza y Carrasco, 2019) y se ha tratado de instrumentar estéticamente como una estrategia de apaciguamiento social e integración de las minorías, la diversidad y variabilidad cultural y el contacto a través del diálogo y buenas maneras, pero sin incidir en las condiciones económico-políticas de la propia desigualdad, sin llegar a políticas concretas y eficaces que impugnen esas desigualdades y exclusiones (Ramírez, 2011). Una verdadera interculturalidad, tendría que de-colonizar los sistemas categoriales, representacionales, interpretativos para crear una nueva relación sistema humano/entorno (Ramírez, 2011).

${ }^{35}$ Desde la interculturalidad en salud no se trata solo de reconocer la diferencia entre diversas formas de atención o diferencias culturales entre la población con respecto a la enfermedad y la salud, sino de evidenciar y enfrentar los abordajes hegemónicos con intereses políticos y económicos (Eroza y Carrasco, 2019) que subyacen en el diálogo de saberes y la prevención y atención a la salud de la población desde cualquier forma de atención en todo contexto social.
}

EntreDiversidades. Revista de Ciencias Sociales y Humanidades, Vol. 9, Núm. 1 (18), enero-junio 2022. Páginas: 247-271 ISSN-e: 2007-7610. https://doi.org/10.31644/ED.V9.N1.2022.A10 
El área encargada al respecto es el Departamento de Enseñanza e Investigación, que tan solo se responsabiliza de realizar cursos de capacitación al personal interesado — no son obligatorios-, por lo que difícilmente se llevan a cabo; de hecho, se desconoce su existencia. Se observan también letreros e información al público sobre campańas de salud en tsotsil y tseltal, instalados en diversas zonas del hospital, así como los lineamientos de interculturalidad. No obstante, según la encargada del departamento, estos no competen al programa de interculturalidad interno, sino que son parte de una política de la SSA estatal. Dicha institución exige al hospital contar con empleados hablantes de estas lenguas, lo cual no ocurre en la práctica, por lo que, para efectos de traducción, se le pide asumir dicho rol al personal de enfermería, vigilancia e intendencia, o a familiares de los pacientes indígenas. ${ }^{36}$

Eroza y Carrasco (2019) consideran que son necesarios estudios de largo aliento para documentar y entender, por ejemplo, el sentido más amplio y profundo de las prácticas de salud de los pueblos indígenas. A su vez, Magaña (2016) considera que un modelo intercultural debería enriquecerse para constituirse en un fuerte sustento para el trazado de políticas en salud dirigidas a población rural, marginal y pobre, no solamente indígena.

Como parte de los lineamientos interculturales, se enuncia hoy día el reconocimiento de las medicinas alternativas y complementarias, como la acupuntura. Sin embargo, una experiencia narrada, muestra limitantes, relativas, por ejemplo, a la vida institucional para introducir estas terapéuticas dentro del sistema de salud oficial; tuvo lugar en el Hospital de Las Culturas. Durante 2010, una médica anestesióloga organizó un diplomado de acupuntura dentro del hospital, en el que participaron 10 personas que laboraban allí y en el Hospital de la Mujer. Realizaron prácticas con pacientes internos y de consulta externa. ${ }^{37} \mathrm{Al}$ respecto, refirió que, aunque el director en turno apoyó este proyecto, tuvieron disputas con personal médico, de enfermería y con algunos departamentos administrativos que no validaban la acupuntura. Aunado a ello, al solicitar a la SSA estatal un área para la atención al dolor, en donde el requisito era incluir a personal del hospital especialista en acupuntura, la SSA no le dio seguimiento y nunca lograron abrir el área.

\section{Casas Maternas}

Otro proyecto que forma parte de la política de interculturalidad en Chiapas es el de las Casas Maternas ${ }^{38}$ a cargo de parteras indígenas, capacitadas por el Sector Salud. Una de ellas se encuentra

\footnotetext{
${ }^{36}$ Información obtenida a través de observación y entrevistas al personal del Hospital de Las Culturas, entre diciembre y enero de 2021.

${ }^{37}$ Entrevista a la médica anestesióloga que lleva laborando 30 años en el hospital y a un empleado del área de planeación y estadística; ambos participaron en el diplomado de acupuntura y son acupuntores actualmente.

${ }^{38}$ Desde el año 2002, a través de la coordinación de Salud de la Mujer de la Jurisdicción Sanitaria no. II (ahora Distrito no. II), en un programa llamado "Desarrollo Integral de Salud para los Pueblos Indígenas" (DISPI), se trabajaba con la finalidad de reducir las tasas de mortalidad materna e infantil; hermanado a un programa federal que se creó en el año 2001, "Arranque parejo en la Vida", en el que el objetivo era atender a la mujer durante el embarazo, en el parto, como puérpera y al recién nacido, con el mismo nivel de atención a mujeres en zonas urbanas y rurales. Una de sus estrategias fue la creación de las Posadas o Casas AME a nivel federal, para la atención materno infantil, que en Chiapas se denominan Casas Maternas. En la actualidad, el programa "Componente Comunitario de Salud
}

EntreDiversidades. Revista de Ciencias Sociales y Humanidades, Vol. 9, Núm. 1 (18), enero-junio 2022. Páginas: $247-271$ ISSN-e: 2007-7610. https://doi.org/10.31644/ED.V9.N1.2022.A10 
instalada dentro del Hospital de Las Culturas. Comenzó a operar cuando fue inaugurado el Hospital en 2010 y se denominaba Área de Medicina Tradicional, pero por dificultades, no del todo claras, la clausuraron en 2015. ${ }^{39}$ Sin embargo, mediante la resistencia y lucha de las parteras con apoyo de instancias no gubernamentales la reabrieron un año después, aunque bajo la supervisión directa de personal comisionado de la Jurisdicción Sanitaria no. II. En el año 2018 la denominaron Casa Materna de San Cristóbal de Las Casas. Al prestar servicios parteras tradicionales junto a personal de la SSA, incide en relaciones de poder desiguales, falta de diálogo entre saberes y sometimiento a las normas institucionales de salud, lo que impide cumplir los objetivos de interrelación de las políticas interculturales (Gómez, 2017; Ruíz, 2018).

La interacción entre parteras tradicionales y la medicina institucional sigue siendo difícil debido a las racionalidades disímiles, una relación de poder mediada por posicionamientos desiguales y una lógica laboral institucional vertical, que ni siquiera confiere a las parteras el estatus de trabajadoras dignas de contar con un salario (Gómez, 2017; Ruíz, 2018).

Una porción de parteras de la zona Altos Tseltal-Tsotsil está incorporada a programas de interculturalidad en salud, pero bajo una lógica de integración y dominación por parte del sistema de salud oficial. Si bien ellas conforman el sector más cooptado por el Sector Salud, más que unificarlas, la persecución y condicionamiento para ejercer sus prácticas a través de capacitaciones ha favorecido el divisionismo entre ellas; mientras que algunas se han integrado a los proyectos como las Casas Maternas, otras se han resistido y luchado, como es el caso de las parteras de la OMIECH. Asimismo, en algunos contextos indígenas de la región, por ejemplo, San Juan Chamula, las mujeres desconfían de las parteras acreditadas por las instituciones, además de que allí suelen ser las parteras pertenecientes a círculos familiares, como madres, tías o abuelas, quienes atienden los procesos reproductivos de las mujeres (Eroza, Morales y Meneses, 2017).

Las parteras siguen siendo una buena opción para las mujeres, principalmente en los contextos rurales, porque buscan el parto natural, pero, actualmente suelen recurrir a instancias médicas alópatas, públicas o privadas, en caso de emergencia obstétrica (Morales, 2021).

\section{Universidad Intercultural}

Por otro lado, los proyectos de interculturalidad no solo han operado en espacios clínicos y hospitalarios, también se han dirigido a la formación académica de médicos interculturales por demás, fallidos - en proyectos de universidades con este enfoque, como la Universidad Intercultural de Chiapas (UNICH), que en 2013 contaba con la carrera de Medicina con Enfoque Intercultural (MEI), pero por condicionantes aparentemente curriculares, aunque en el fondo político-económicas, se modificó a Médico Cirujano (Magaña, 2016). No solo se trataba de la

de la Mujer” es el que se encarga de las Casas maternas. Hasta el 2018 había cuatro Casas funcionando en la zona Altos: San Cristóbal de Las Casas, Chamula, Zinacantán y Larráinzar (Ruíz, 2018).

${ }^{39}$ De manera similar, en 2011, La Casa materna de San Andrés Larrainzar permanecía casi siempre cerrada, pues por lo general las mujeres no se interesaban en utilizarla ni siquiera para atender su embarazo, por lo mismo, la mujer encargada de abrirla casi nunca estaba, de modo que cuando alguien decidía acudir a tratarse, pues no había servicio (Observaciones en campo, 2011).

EntreDiversidades. Revista de Ciencias Sociales y Humanidades, Vol. 9, Núm. 1 (18), enero-junio 2022. Páginas: $247-271$ ISSN-e: 2007-7610. https://doi.org/10.31644/ED.V9.N1.2022.A10 
puesta en marcha de un programa trazado con un perfil intercultural, sino de impulsar relaciones interinstitucionales para intervenir en las acciones de salud, en un contexto pluricultural y con diversos intercambios interétnicos.

\section{El ámbito privado: biomédico y alternativo}

Los servicios biomédicos privados son también una opción significativa en la región, la cual se ha ido incrementando. En la actualidad, existen cinco hospitales privados en la cabecera regional, a los que asiste población diversa capaz de afrontar los costos de su atención. ${ }^{40}$

A la par, se encuentran los consultorios privados en medicina general, especialidades médicas varias, odontología, nutrición, psicología, laboratorios de análisis clínicos, rayos X y de estudios médicos especializados y servicios de enfermería, que dan cuenta de una diversificación de costos — algunos se encuentran subrogados a los servicios de salud públicos-.

Dada la estratificación socioeconómica que por diversos factores se ha desarrollado en la zona Altos, los sectores más beneficiados económicamente han tendido a optar por la utilización de servicios privados. Hemos observado, por ejemplo, que, si bien existen restricciones de acceso de familiares a los espacios hospitalarios, a sabiendas de que los pacientes indígenas representan grandes entradas económicas, dichas restricciones se relajan. Por el mismo motivo, se advierten prácticas médicas tendientes a prolongar la hospitalización de los pacientes.

Asimismo, dada la numerosa presencia de personas provenientes de otras regiones de México y de diversos países, abundan, sobre todo en San Cristóbal de Las Casas, servicios privados de Medicinas Alternativas y Complementarias, clasificadas así por la OMS (2002). ${ }^{41}$ Sus sedes se ubican mayormente en zonas urbanas.

En la ciudad de San Cristóbal se pueden encontrar, además, las Casas de Partos, donde laboran parteras profesionales. Son espacios como una casa habitación, en las que se otorgan atención al embarazo, parto, puerperio, entre otros. Su objetivo es recrear una atmósfera confortable, relajante y segura para mujeres embarazadas y sus acompańantes (Sánchez, 2016) desde la perspectiva del parto humanizado, al igual que en las Casas Maternas. No obstante, de acuerdo con Morales (2021), en estos contextos, el trato humanizado que promueven muchas veces no se encuentra al alcance de toda la población por los altos costos de atención, y aunque las mujeres buscan la desmedicalización, terminan por ser medicadas.

\footnotetext{
${ }^{40}$ El Hospital y Centro Médico San Cristóbal (HOSCEM), Hospital de Caridad A. C., Sanatorio Ornelas, Sanatorio Dr. Bonilla y Hospital Colonial.

${ }^{41}$ Según la OMS, las medicinas alternativas son aquellas que tienen raíces en los conocimientos de salud y enfermedad de las diferentes medicinas tradicionales chinas, hindúes, japonesas y de otras culturas del mundo, como: acupuntura, digitopuntura, ayurveda, reflexología, tai chi, yoga, reiki, etc. Las medicinas complementarias se consideran derivadas del sistema biomédico y no forman parte de la tradición de un país, algunas de las que se ofrecen son: homeopatía, quiropráctica, terapias bioenergéticas, radiestesia, entre otras. Merece la pena profundizar en otros estudios esta categorización de la OMS.
}

EntreDiversidades. Revista de Ciencias Sociales y Humanidades, Vol. 9, Núm. 1 (18), enero-junio 2022. Páginas: 247-271 ISSN-e: 2007-7610. https://doi.org/10.31644/ED.V9.N1.2022.A10 
Otras opciones privadas son las clínicas de rehabilitación para adicciones. Una de ellas se encuentra en San Cristóbal, "Volver a Vivir Libre, A.C.", que ofrece sus servicios con el mensaje de ayudar a "alcohólicos, drogadictos e ingobernables", fundada en enero del año 2000 (Volver a Vivir Libre, A. C., 2021).

Se puede decir, en términos generales, que la diversidad de opciones privadas es amplia, no todas se incluyen aquí, su variedad se da en función de formas, lugares, personas y costos.

\section{Prácticas tradicionales y religiosas}

Las concepciones y prácticas indígenas en torno a la salud y la enfermedad son una forma de atención en la zona Altos Tsotsil-Tseltal que combina elementos prehispánicos mayas con elementos religiosos católicos configurados desde el virreinato, son las concepciones y prácticas indígenas en torno a la salud y la enfermedad. Persisten entre esta población tradiciones relativas a la atención a la salud como parte de su cultura en un sentido amplio, las cuales aluden a dinámicas sociales, a creencias religiosas, a valores y a visiones en torno a la persona. Concepciones y prácticas que conviven con otras tradiciones y referentes médicos que se brindan de forma diferenciada, ya sea en un espacio particular o en centros donde se ofrecen también las medicinas alternativas. ${ }^{42}$

Los grupos religiosos son también notables en materia de prácticas de salud, porque promulgan la adhesión a los cultos que profesan como premisa para mantener o recuperar la salud.

Un ministro católico de San Cristóbal de Las Casas ${ }^{43}$ nos habló de dos sacramentos considerados de sanación; la Confesión y la Unción de los enfermos. Respecto al segundo, refirió que los adeptos acuden a la casa de una persona enferma o celebran misas especiales para sanarla. Las iglesias no católicas también operan como redes de apoyo en materia de salud, no solo en lo relacionado con recursos, también en lo que respecta al apoyo emocional. La curación desde la religiosidad resulta, así, un recurso que articula la atención física y emocional del padecer.

En términos generales, sin embargo, las congregaciones religiosas han creado espacios para la atención a la salud, en los que reciben a personas sin que el requisito sea siempre asumir sus preceptos, aunque enfocan parte de sus servicios en prácticas de la religión que profesan. Un ejemplo de ello son el Hospital de Caridad, dirigido por monjas católicas en San Cristóbal, y el centro de rehabilitación para adicciones CIF BETESDA (Centro de Integración Familiar). ${ }^{44}$

\footnotetext{
${ }^{42}$ Por ejemplo, La Montańa, ubicado en la zona sur de la ciudad de San Cristóbal, espacio terapéutico fundado por un terapeuta indígena conocido como Don Lauro, donde brindan ritos indígenas, algunas terapias alternativas, retiros, talleres y recorridos de montaña, entre otras opciones. Y Nahual-ha, ubicado en la zona poniente de la misma ciudad, fundado por una terapeuta alternativa de la ciudad de México, y se ofrecen opciones de terapias indígenas, alternativas y spa, además de formaciones académicas en diversas opciones alternativas y complementarias.

${ }^{43}$ Información obtenida con un ministro de una iglesia católica en San Cristóbal de las Casas. Refiere que los ministros pueden ser cualquier persona, hombre o mujer, que quiera apoyar en las parroquias. Estos reciben una formación de dos años por un sacerdote asignado para ello y les confieren acciones ministeriales litúrgicas.

${ }^{44}$ Información obtenida en entrevista a una psicóloga que presta servicio social en este centro, y menciona: "Este centro es particular, no pertenece a ninguna Iglesia, aunque los directivos son cristianos y se enfocan mucho a las cosas de Dios, reciben a cualquier persona, y más o menos duran de 4 a 6 meses aproximadamente en tratamiento" (febrero, 2021).
}

EntreDiversidades. Revista de Ciencias Sociales y Humanidades, Vol. 9, Núm. 1 (18), enero-junio 2022. Páginas: $247-271$ ISSN-e: 2007-7610. https://doi.org/10.31644/ED.V9.N1.2022.A10 
No se puede soslayar, empero, que, en varios casos, el cometido de ganar y asegurar adeptos conlleva discursos y prácticas que versan en convencer a las personas enfermas de que el mantenimiento y restablecimiento de la salud dependen del hecho de abrazar la fe que cada comunidad religiosa mantiene.

Por lo demás, muchas personas indígenas acuden a iglesias, templos y ermitas, ya sea en sus comunidades o en localidades urbanas, para ser partícipes de estas diferentes congregaciones religiosas —a iglesias católicas donde se realizan misas para la unción de los enfermos o a iglesias protestantes para realizar sesiones de culto tendientes a tratar problemas de salud-. Aunque además de lo referido por el ministro católico, los miembros de cultos protestantes también suelen trasladarse al hogar de las personas enfermas a fin de emprender rezos en favor de su salud. ${ }^{45}$

\section{La medicalización a través de las farmacias}

Una opción a la que un amplio sector de la población acude, quizá mayormente indígena, es la utilización de farmacopea proveída en farmacias. Tiene lugar ya sea mediante la automedicación como a partir de la medicación prescrita por los dueńos o empleados de las farmacias.

En Chiapas, algunas comunidades contaban en los noventa con farmacias de tipo comunitario, que se establecieron en puestos médicos del INI y eran manejadas por un comité de salud o por promotores de salud que prescribían tratamientos (Freyermuth, 1993). La presencia de farmacias en las comunidades de los Altos se gestó a partir de los promotores en salud, como se aprecia, originalmente formados a partir de las políticas en salud del INI y posteriormente de otras políticas institucionales. ${ }^{46}$ Sin embargo, al quedar desempleados al final de diferentes periodos políticos, algunos abrieron sus farmacias. Lo interesante en términos de interculturalidad es que desde entonces prescriben desde su propia lógica, por ejemplo, el consumo de vitaminas que al favorecer el mantenimiento de un cuerpo activo promueve la creencia de que lo mantiene saludable, o bien la clasificación de medicinas en función de sus calidades frías o calientes (Holland, 1963; Harman, 1969; Eroza, 2016).

En San Cristóbal de Las Casas y demás municipios de la región, también hay farmacias donde se ofrece consulta como una estrategia mercadotécnica tendiente a promover la compra de la farmacopea que ofertan, incluyendo medicinas naturistas. Pertenecen a dueños particulares y a cadenas de farmacias, tanto locales como nacionales, que compiten entre sí vendiendo medicamentos alópatas y herbolarios. Aunque también se puede hablar de cadenas de farmacias con venta exclusiva de medicamentos naturistas.

\footnotetext{
${ }^{45}$ Hemos constatado que, en ciertos casos, los pastores protestantes también utilizan la retórica religiosa para convencer a las personas de que accedan a consumir farmacopea prescrita por médicos.

${ }^{46}$ Medida que aún promueven organizaciones como las de la sociedad civil en la formación de nuevos promotores.
}

EntreDiversidades. Revista de Ciencias Sociales y Humanidades, Vol. 9, Núm. 1 (18), enero-junio 2022. Páginas: 247-271 ISSN-e: 2007-7610. https://doi.org/10.31644/ED.V9.N1.2022.A10 


\section{Prácticas de autocuidado y grupos de autoayuda}

Ante los padecimientos, los sujetos no solo recurren a las prácticas de distintos actores en atención a la salud, sino que es común la utilización de recursos terapéuticos propios, prácticas que se trasmiten de generación en generación, como, por ejemplo, recetas caseras de alimentos naturales, elaborados por quienes cuidan a las personas enfermas. ${ }^{47}$

Es también habitual en la región asistir a grupos de autoayuda para la recuperación de problemas emocionales y/o adicciones, como Alcohólicos Anónimos (AA), Neuróticos Anónimos y los grupos de $4^{\circ}$ y $5^{\circ}$ paso, que se dirigen al apoyo mutuo a través de sus propios testimonios y se mantienen con cuotas voluntarias de sus miembros..

De acuerdo a un coordinador de AA, ${ }^{48}$ en San Cristóbal existen alrededor de cuarenta y cinco grupos, tres en Oxchuc, uno en Chamula y uno más en Huixtán. Otros grupos son los llamados de $4^{\circ}$ y $5^{\circ}$ paso, basados en el cuarto y quinto paso de los doce pasos de $\mathrm{AA},{ }^{49}$ pero sin pertenecer a ellos. Además de San Cristóbal, estos también se encuentran en el municipio de Tenejapa, igualmente perteneciente a la región.

\section{La diversidad en el mismo contexto}

En materia de salud, históricamente han participado iglesias católicas y no católicas, órganos representantes de políticas de atención a grupos indígenas, organizaciones de parteras y médicos indígenas, parteras tradicionales y médicos indígenas independientes, organizaciones civiles, instituciones del sector salud, agencias internacionales, hospitales y servicios de salud privados, servicios particulares de parteras profesionales, medicinas alternativas y complementarias, grupos de autoayuda y las prácticas de autocuidado propias de la población. Aunque también las empresas productoras y distribuidoras de multivitamínicos resultan dignas de mención.

Por lo mismo, no existe una sola mirada para entender y atender la salud y la enfermedad en la zona. La diversidad de opciones de atención se ha gestado a través de procesos históricos propiciados por el influjo de dinámicas culturales, sociales, políticas y económicas, así como por la interacción entre escalas locales y globales. Pero la construcción de esta región sanitaria, no

\footnotetext{
${ }^{47}$ Como lo mencionan pacientes del Hospital de Las Culturas en los siguientes testimonios: "Salió que tenía yo anemia; y tres meses comí pura res y verdura [...] esos tres meses comí cuando estaba yo en la Casa de la Quinta: nabito, la acelga, la espinaca, puro hígado de res, pero ya no lo puedo ver [...] Desde agosto me sentí mal, tomé plantas y plantas y plantas [...] mi hermanita me hacía cuando estaba yo en mi casa jugo de naranja con betabel" (Entrevista a paciente, Hospital de Las Culturas, enero 2021). "[...] al menos yo no he buscado otro tipo de tratamiento, de repente, no siempre, mi mamá me los da, pero ella sí ha buscado cosas naturales y le ha funcionado" (Entrevista a paciente, Hospital de Las Culturas, enero 2021).

${ }^{48}$ Coordinador del grupo de AA "Camino a la Verdad", y ex miembro del comité del Distrito II de AA de San Cristóbal de Las Casas del área geográfica sur. Refiere que sesionan dos horas diarias de lunes a viernes, y anualmente tienen una semana de encuentro por aniversario y una sesión de información pública que en los últimos años lo hacen a través de la radio o de canales de Facebook, para expandir su mensaje en varios lugares.

${ }^{49}$ El cuarto paso es "Sin temor hicimos un minucioso inventario moral de nosotros mismos" (AA, 2021a: 40) y el quinto paso es "Admitimos ante Dios, ante nosotros mismos y ante otro ser humano la naturaleza exacta de nuestros defectos" (AA, 2021b: 53). Comienzan con siete juntas de preparación para luego realizar los pasos en un retiro espiritual (Unidad 12 pasos, 2019).
}

EntreDiversidades. Revista de Ciencias Sociales y Humanidades, Vol. 9, Núm. 1 (18), enero-junio 2022. Páginas: $247-271$ ISSN-e: 2007-7610. https://doi.org/10.31644/ED.V9.N1.2022.A10 
solo ha sido y es protagonizada por los proveedores de las diferentes formas de atención, también por los destinatarios de las mismas, quienes, lejos de limitar su búsqueda de salud a una sola, de muchas formas transitan al menos por varias de ellas, en función del curso de sus experiencias del padecer.

\section{Articulación de opciones de atención a la salud}

En este apartado exponemos lo que las personas de los dos estudios inicialmente citados refieren respecto a la diversidad de opciones a las que recurrieron, las cuales tienden a articular a partir de la lógica que trazan en su trayectoria como enfermos y, precisamente, de las opciones de atención que les brinda el contexto regional. Por tanto, son las personas enfermas quienes pueden hablar de qué lógica incide en su transitar por las diversas opciones de atención a la salud.

Un referente importante que mencionaron personas tsotsiles y tseltales respecto a la atención de sus problemas de salud es la consulta con los médicos indígenas desde un marco interpretativo de la enfermedad/infortunio, el cual amplifica la mirada en torno al sufrir (Eroza, 2016).$^{50}$ En algunas ocasiones buscan atención biomédica antes de asistir con los médicos indígenas, pero si los malestares persisten, gradualmente se van incorporando lecturas de orden sociocultural en las que por lo general predominan ideas acerca de retribución divina o bien de brujería (Eroza, 2016).

Los médicos indígenas se dividen de acuerdo con su especialidad, los j-iloletik y poxtawanejetik, ${ }^{51}$ el Tzac 'bak (huesero), el Ac'vomol (hierbero) y la Jve't'ome o chilil 52 (partera) y se desempeñan dentro de su localidad y en otros municipios, como San Cristóbal de Las Casas, donde se encuentra el Museo de Medicina Maya de OMIECH, en el cual también comercian medicamentos elaborados con herbolaria. A este Museo no solo acude gente de comunidades, también mestizos e indígenas residentes de las cercanías del museo (Ruíz, 2018).

Entre los recursos terapéuticos que brindan, además de productos herbolarios, se encuentran: rituales de protección y conservación de la salud, tales como limpias con albahaca, flores y huevos y quema de velas; rezos frente a altares de imágenes católicas, en cuevas y dentro de las iglesias; sobadas para curar fracturas y zafaduras de huesos. Muchos terapeutas indígenas son al mismo tiempo hueseros, rezadores, parteros y ritualistas.

Además de la de atención con médicos indígenas, hay referentes del uso de medicinas basadas en herbolaria procedentes de otras latitudes y empresas especializadas en la venta de multivitamínicos como Omnilife y Herbalife. En relación con estas últimas, hay personas, en su mayoría indígenas, que distribuyen su catálogo en las comunidades habiendo sido antes capacitados para emprender dicha actividad e inclusive prescribir los productos, los cuales son visualizados como recursos para superar todo tipo de padecimientos, perspectiva que parece

\footnotetext{
50 "La dialéctica entre ideas relativas a castigo divino, brujería y a concepciones espirituales y de su correlación con la experiencia cotidiana, vivida y/o percibida, de la vida social” (Eroza, 2016: 97).

${ }^{51}$ Terapeutas indígenas tsotsiles y tseltales, respectivamente, que basan sus prácticas de diagnóstico y curación a través de rezos y oraciones, sueńos y el pulseo.

${ }^{52}$ Partera en tsotsil y tseltal, respectivamente.
}

EntreDiversidades. Revista de Ciencias Sociales y Humanidades, Vol. 9, Núm. 1 (18), enero-junio 2022. Páginas: 247-271 ISSN-e: 2007-7610. https://doi.org/10.31644/ED.V9.N1.2022.A10 
tener como trasfondo la idea de que energizan el cuerpo y ayudan a mantenerse activo, signo inequívoco, para muchos indígenas, de un cuerpo sano.

También se observa en días de plaza de las comunidades a vendedores indígenas de hierbas medicinales, que las ofertan para el tratamiento de muchos padecimientos (Eroza, 2016). En esta práctica también subyacen criterios relacionados con lo que se considera un cuerpo sano, así como la dualidad frío/calor relativa a los tipos de enfermedades y a los propios recursos terapéuticos.

Hasta aquí, se han mencionado las opciones que las personas indígenas tienen a su disposición en su contexto inmediato y las referentes a procesos socioculturales amplios. Hoy día, sin embargo, sin que las personas se desvinculen de estas prácticas, resulta también frecuente el acercamiento a las instituciones del Sector Salud, en primer y segundo nivel de atención, muchas veces a través de programas asistenciales como "PROSPERA", ${ }^{3}$ que a partir de 2021 se denomina "Programa Nacional de Becas para el Bienestar". En parte, ello se debe al beneficio económico que conlleva, lo que resulta un incentivo para utilizar los servicios médicos institucionales. No obstante, hay quienes lo hacen por decisión propia ante la necesidad de atender los aspectos de su padecimiento, lo que no quiere decir que este tipo de alternativa inhiba la utilización de las otras, aunque sí pone de manifiesto que en la diversidad de prácticas también interviene la heterogeneidad sociocultural de la que da cuenta el contexto regional. Por consiguiente, en los complejos y variados procesos de búsqueda de salud median múltiples dimensiones causales.

De manera parcial, las trayectorias terapéuticas de la población son influenciadas por el curso de sus padecimientos, es decir, si en un momento dado alguna opción de atención ha contribuido o no a mejorar su salud. No obstante, al menos en lo que concierne a las personas indígenas entrevistadas, una racionalidad que también interviene se refiere a la idea de que la enfermedad constituye una de las muchas manifestaciones del infortunio propio e incluso familiar. De tal suerte, su búsqueda de salud se guía en gran medida a partir de dicha lógica, lo cual constituye una razón más por la cual las concepciones y prácticas en salud por parte de estas poblaciones no pueden pensarse vis a vis con la epistemología ni con la práctica biomédica.

En lo que compete a las trayectorias del padecer de pacientes que han acudido al Hospital de Las Culturas (población indígena y no indígena), las primeras respuestas ante su padecimiento están relacionadas con formas de autocuidado, de acuerdo con los recursos que brinda su entorno. Es decir, ante los primeros malestares, quienes los padecen se dirigieron en primera instancia a las personas cercanas, como vecinos, conocidos y familiares. En primer término, si se trata de malestares menores, los recursos terapéuticos que suelen entrar en escena son remedios de diversa índole (Ruíz, 2021).

Refirieron utilizar, por ejemplo, plantas medicinales preparadas como infusiones y/o compresas, alimentos con propiedades curativas como nopal, berro, punta de chayote, nabo, acelga, entre otros. Como anticipamos, una de las lógicas que subyace respecto a la utilización de este tipo de recursos fue referida por dos pacientes, una mujer de origen tseltal y otro no indígena,

\footnotetext{
${ }^{53}$ El programa de Inclusión Social PROSPERA tenía el objetivo de articular y coordinar la oferta institucional de programas y acciones de política social, incluyendo aquellas relacionadas con el fomento productivo, generación de ingresos, bienestar económico, inclusión financiera y laboral, educación, alimentación y salud, dirigida a la población que se encuentre en situación de pobreza (Secretaría de Bienestar, 2016).
}

EntreDiversidades. Revista de Ciencias Sociales y Humanidades, Vol. 9, Núm. 1 (18), enero-junio 2022. Páginas: 247-271 ISSN-e: 2007-7610. https://doi.org/10.31644/ED.V9.N1.2022.A10 
quienes hablaron de la eficacia de los recursos, calientes o fríos, que utilizaron en función del contra efecto de la calidad fría o caliente de sus padecimientos.

También, fue posible identificar los recursos de los propios sujetos a nivel de sostenimiento del yo, del cuerpo como espacio vivido ${ }^{54}$ en relación con el padecimiento y el restablecimiento de la salud, como la fe en entes sagrados y/o el buen comportamiento que debe observarse. Veamos algunos ejemplos:

[...] lo vi cuando mi papá, él estuvo dos años enfermo, sentado [llora] él ve, él oye, él platica muchas historias, bonitas historias, por eso me recuerdo [...] pero me contó muchas cosas, y lo estoy viendo ahorita: la vida de la Madre tierra, el abuelo Sol, el viento, el agua (Entrevista al hermano de una paciente, diciembre 2020).

Alguien dijo que no iba yo a salir adelante, un montón de cosas que decían, pero gracias a dios estoy mejor, ya camino sin bastón de aquí hasta el taller, con mi nieto le digo: "vámonos a caminar hasta allá atrás", y nos vamos juntos ya se me hace más fácil todo (Entrevista a expaciente del Hospital de Las Culturas, febrero 2021).

Algo me dice mi cuerpo, no lo he entendido, si ya avancé mucho [...] por eso a pesar de todos los accidentes confío en encontrar mi crecimiento interno (Entrevista a expaciente del Hospital de Las Culturas, febrero 2021).

También el ánimo y la motivación de sanar por su familia es un recurso de sostenimiento del yo:

Pero lo que me motiva a salir adelante principalmente es la familia, mis hijos (Entrevista a paciente del Hospital de Las Culturas, diciembre 2020).

Ante las distintas instancias y actores de la biomedicina, las personas refirieron que no solo acceden a la atención pública, sino a los servicios privados ya mencionados. Si bien algunas consideran que estos son más rápidos en comparación con el sistema público de salud, esta opción se convierte en una transacción de oferta y demanda, con costos elevados, ${ }^{55}$ que muchas veces son asumidos como necesarios de cubrir ante alguna problemática de salud dada.

Las terapias alternativas y/o complementarias son actualmente en esta zona una opción cada vez más socorrida, aunque la utiliza en mayor medida la población foránea y la que reside en San Cristóbal, también la población indígena busca eventualmente atención en dichos ámbitos.

\footnotetext{
${ }^{54}$ Espacio de representación donde se refleja subjetividad, es un espacio propio que construye cada sujeto en relación con los demás, se estructura una nueva dimensión material y mental en el individuo conformando un proceso dinámico con el tiempo y generando nuevos valores actitudes e intereses (Lefebvre, 1974).

${ }^{55}$ En una entrevista con el familiar de una paciente del Hospital de Las Culturas, ella mencionó que antes de buscar el servicio público asistieron con un médico particular que les ofreció transfusión sanguínea para su hermana, pero les cobraba $\$ 5000$ la noche, a lo cual no pudieron acceder. Otro paciente refiere que prefiere realizarse sus estudios de laboratorio de manera privada, aunque gaste mucho, porque así no tiene que enfrentarse a la pérdida de tiempo y molestias ante ciertas actitudes del personal de salud pública.
}

EntreDiversidades. Revista de Ciencias Sociales y Humanidades, Vol. 9, Núm. 1 (18), enero-junio 2022. Páginas: $247-271$ ISSN-e: 2007-7610. https://doi.org/10.31644/ED.V9.N1.2022.A10 
Rocío Marisela Ruiz Ruiz

José Enrique Eroza Solana

Jorge Magaña Ochoa
Configuración de la región sanitaria de la zona Altos Tsotsil-Tseltal: panorama...

En lo tocante a los servicios públicos, como el Hospital de Las Culturas, en la mayoría de los casos fue el último lugar en acudir, hasta que el mal fue de gravedad, pero el seguimiento clínico al padecimiento resultó difícil porque el Hospital no contaba con todos los servicios ni todas las especialidades y/o porque se tienen que pagar los medicamentos o los estudios en servicios privados. El hospital público deviene así en el espacio en el que se atiende principalmente la urgencia:

Vine acá con mis análisis, lo trajimos el resultado; por eso me pasaron, si no, no me pasaban, como me miraron caminando, pues. Parece que tenía yo 1.0 de hemoglobina, por eso recibí 6 unidades; por eso quieren 8 o 10 donadores [...] Cuando llegué al hospital, en la tarde, el muchacho que me atendió me dijo: “¿y por qué hasta ahorita señora?”. "Es que nadie me quería firmar, nadie me quería acompañar para que yo entrara aquî". "Ay señora, te pasas”, me dijo el muchacho. No podía yo hablar porque me agitaba, y temblando de frío (Entrevista a paciente del Hospital de Las Culturas, diciembre 2020).

Antes yo iba en particulares, bueno no con pancreatitis, sino con algunas otras complicaciones, iba con diferentes doctores, pero cuando es de pancreatitis sí vengo acá, aquí las tres veces que me han dado pancreatitis aquí las tres veces me han atendido (Entrevista a paciente del Hospital de Las Culturas, diciembre 2020).

Pero de los más graves y que caí en el Hospital de las Culturas, bueno que caímos, fue un accidente en moto que tuvimos con mi papá [...] Ya mandaron a llamar a las ambulancias y no sé quién dio la orden o si las ambulancias solitas te llevan al Hospital de las Culturas, pero nos llevaron ahí a mi papá y a mí, yo creo que fue como de emergencia. Y resulta que en el Hospital de las Culturas no tenían medicamentos para el dolor, era una cosa, así como, si mal no recuerdo me curaron, y todo sin anestesia y sin medicamento para el dolor (Entrevista a expaciente del Hospital de Las Culturas, febrero 2021).

Entre muchas personas, indígenas principalmente, persiste el imaginario de que en los hospitales las personas llegan a morir, lo que guarda relación, por un lado, con lo referido líneas arriba en el sentido de que a menudo los enfermos son llevados allí ya en un estado de gravedad, pero también, por otro lado, con la negligencia médica que persiste en dichos contextos, en la cual no solo media el burocratismo, también una agenda racista y clasista de profundas raíces históricas que excluye, y en el mejor de los casos, infantiliza, a la población indígena.

Las experiencias dejan ver el sentido que tiene para los pacientes, como sujetos/agentes, su propio proceso en la recuperación de su enfermedad, y el espacio hospitalario suele ignorar el hecho de que los sujetos articulan las diversas prácticas a las que han recurrido mediante una lógica propia. Por tanto, una región sanitaria no solo la configuran las formas de atención, sino los usuarios de estas con sus propias decisiones, a menudo mediadas por vicisitudes y referentes socioculturales, que, por vivir en un contexto diverso, y sobre todo por el carácter de sus búsquedas de salud, son ya de por sí interculturales.

EntreDiversidades. Revista de Ciencias Sociales y Humanidades, Vol. 9, Núm. 1 (18), enero-junio 2022. Páginas: $247-271$ ISSN-e: 2007-7610. https://doi.org/10.31644/ED.V9.N1.2022.A10 
Rocío Marisela Ruiz Ruiz

José Enrique Eroza Solana

Jorge Magaña Ochoa
Configuración de la región sanitaria de la zona Altos Tsotsil-Tseltal: panorama...

\section{Reflexiones finales}

Con la finalidad de comprender los procesos de salud/enfermedad en la zona Altos Tsotsil-Tseltal, hemos documentado y analizado el proceso de construcción de una Región Sanitaria que se ha ido gestando con el paso del tiempo, a través de estructuras sociales y procesos socioculturales, políticos y económicos, mediante relaciones desiguales y visibles de poder y disputas ideológicas, a la vez que intersubjetivas e identitarias, todo lo cual da cuenta de un flujo continuo de construcción y reconstrucción mediante la interacción de diversos actores sociales.

Los procesos sanitarios de esta región, de tal suerte, dan cuenta de importantes particularidades a pesar de mostrar procesos estructurales comunes respecto a otras regiones. Destaca, empero, el devenir histórico de los indígenas, matizado por la violencia y la inequidad propias de una larga era colonial. Devenir que se sigue expresando en políticas específicas en salud. Aún de manera reciente la amplia y variada presencia de agentes foráneos en la zona no logra superar del todo con sus intervenciones en salud las visiones condescendientes para con las concepciones y prácticas de estas poblaciones.

En el mismo tenor, se puede hablar, como lo han hecho algunos estudios (Menéndez, 1992; Magaña, 2002; Eroza, 2016), de la hegemonía de la medicina científica, a través de prácticas, políticas públicas de salud, e inclusive, desde el ejercicio de la mirada social de los médicos y médicas de las Organizaciones de la Sociedad Civil.

Desde dicha postura, la complementariedad entre prácticas médicas se vislumbra como una meta inalcanzable a lo que quizás se podría aspirar y con ello alcanzar logros significativos. Se trata de abrir espacios de comunicación más horizontales para el logro de un mutuo entendimiento y, por ende, de la posibilidad de negociar mecanismos para solucionar conjuntamente problemáticas de salud.

La amplia diversidad de actores que han participado y participan en la conformación de esta región sanitaria nos revela, sobre todo en el presente, un espacio de atención marcadamente cosmopolita que confiere a las personas y sus padecimientos la posibilidad de transitar por búsquedas de salud que auspician una variedad inagotable, en la que sin duda también participa una profusa imaginación cultural que suele rendir historias insospechadas del padecer y del sufrir.

No ha sido aquí nuestro cometido pronunciarnos en contra de la biomedicina ni su posicionamiento de poder frente a otras concepciones y prácticas relativas a la salud y la enfermedad. Nuestra propuesta versa más bien en invitar a sus representantes a hacer un lado la unilateralidad de su mirada y abrirse al diálogo con las personas que atienden y descubrir el sentido que otorgan a sus males, así como la resistencia que oponen a los mismos. Ello posibilitará una verdadera práctica intercultural en salud.

Por lo demás, entender las visiones y los sentires de las personas en torno a su padecer, nos permite vislumbrar lo significativo de su rol en calidad de partícipes en la construcción de la Región Sanitaria de la zona Altos Tsotsil-Tseltal; junto a los muchos y diversos actores sociales que han estado presentes históricamente de forma individual y colectiva. Y al mismo tiempo, nos parece fundamental resaltar que la interculturalidad en la salud parte de los propios usuarios de las prácticas médicas al articular los diversos recursos terapéuticos a los que recurren en el transitar de su padecimiento.

EntreDiversidades. Revista de Ciencias Sociales y Humanidades, Vol. 9, Núm. 1 (18), enero-junio 2022. Páginas: $247-271$ ISSN-e: 2007-7610. https://doi.org/10.31644/ED.V9.N1.2022.A10 
Rocío Marisela Ruiz Ruiz

José Enrique Eroza Solana

Jorge Magaña Ochoa
Configuración de la región sanitaria de la zona Altos Tsotsil-Tseltal: panorama...

\section{Bibliografía citada}

Alcohólicos Anónimos, AA. (2021a). Cuarto paso [en línea]. Disponible en: https://www.aa.org/ sites/default/files/2021-11/sp step4.pdf (Consultado el 20 de noviembre de 2021).

Alcohólicos Anónimos, AA. (2021b). Quinto paso [en línea]. Disponible en: https://www.aa.org/ sites/default/files/2021-11/sp step5.pdf (Consultado el 20 de noviembre de 2021).

Albet i Mas, Abel (1993). "La nueva geografía regional o la construcción social de la región". Anales de Geografía de la Universidad Complutense [en línea], Núm. 13, pp.11-29. Disponible en: https://revistas.ucm.es/index.php/AGUC/article/view/AGUC9393110011A/31664 (Consultado el 2 de agosto de 2021).

Alianza por la salud, alimentación y nutrición (ASAN) (2020). Directorio de ASAN [Folleto].

Campos Navarro, Roberto (1996). Legitimidad social y proceso de legalización de la medicina indigena en América Latina, Estudios de México y Bolivia. Tesis para obtener el grado de Doctorado en Antropología, Facultad de Filosofía y Letras, Universidad Nacional Autónoma de México. Disponible en: https://repositorio.unam.mx/contenidos/63558 (Consultado el 10 de julio de 2021).

Centro de Atención Contra la Violencia Familiar (CAVIF) (2021). Centro de Atención de La Violencia hacia las Mujeres [Facebook]. Disponible en: https://web.facebook.com/cavif. chiapas/? rdc=1\& rdr (Consultado el 19 de noviembre de 2021).

Eroza Solana, Enrique (2016). El cuerpo como texto y eje vivencial del dolor. Las narrativas del padecimiento entre los tsotsiles de San Juan Chamula. México, D. F., México: Centro de Investigaciones y Estudios Superiores en Antropología Social, Publicaciones de la Casa Chata.

Eroza Solana, Enrique, Magdalena Morales Domínguez y Sergio Meneses Navarro (2017). "Preferencias y tema de decisiones para la atención del embarazo y parto en municipios de Chiapas", en Freyermuth Enciso, María Graciela (coord.). Salud y Mortalidad Materna en México. Balances y perspectivas desde la antropología y la interdisciplinariedad. México, D. F., México: Centro de Investigaciones y Estudios Superiores en Antropología Social, pp. 86-127.

Eroza Solana, Enrique y Carrasco Gómez, Mónica (2019). "La interculturalidad y la salud: reflexiones desde la experiencia”. LiminaR. Estudios Sociales y Humanisticos [en línea], 13 (1), pp. 112-128. doi: https://doi.org/10.29043/liminar.v18i1.725 (Consultado el 20 de marzo de 2020).

Freyermuth Enciso, María Graciela (1993). Médicos tradicionales y médicos alópatas. Un encuentro difícil en los Altos de Chiapas. Chiapas, México: Centro de Investigaciones y Estudios Superiores en Antropología Social Sureste, Organización de Médicos Indígenas del Estado de Chiapas, A.C.

García Álvarez, Jacobo (2006). "Geografía Regional”, en Alicia Lindón y Hiernaux, Daniel (dirs.), Tratado de Geografía Humana. México, D. F., México: Universidad Autónoma Metropolitana, Ixtapala, División Ciencias Sociales y Humanidades, Anthropos Editorial, pp. 44-64.

EntreDiversidades. Revista de Ciencias Sociales y Humanidades, Vol. 9, Núm. 1 (18), enero-junio 2022. Páginas: $247-271$ ISSN-e: 2007-7610. https://doi.org/10.31644/ED.V9.N1.2022.A10 
Gómez García, Irazú (2017). Echar valor. Parteras tradicionales en el contexto biomédico del sector salud. Tesis para obtener el grado de Mestría en Antropología, Programa de Posgrado en Antropología Chiapas, Universidad Nacional Autónoma de México. También disponible en: https://repositorio.unam.mx/contenidos/98249

Harman, Robert Charles (1969). Medical and Social Changes in a Tzeltal Community. Tesis para obtener el grado de Doctorado en Antropología, Universtity Microfilms, Ann Harbor Michigan.

Hogar Comunitario Yach'il Antzetic, A. C. (2021). Información [Facebook]. Disponible en: https://web.facebook.com/mujeresnuevass/? rdc=1\& rdr (Consultado el $20 \mathrm{de}$ noviembre de 2021).

Holland William (1963). Medicina Maya en los Altos de Chiapas; un estudio del cambio sociocultural. México, D. F., México: Instituto Nacional Indigenista.

Jiménez Acevedo, Hilda y Núñez Medina, Gerardo (2016). "El sistema de salud de Chiapas ante la transición demográfica y epidemiológica”. Cuadernos del Centro de Estudios del Desarrollo (CENDES) [en línea], Año 33, Núm. 92, Tercera época, pp. 79-103. Disponible en: http://ve.scielo.org/pdf/cdc/v33n92/art05.pdf (Consultado el 28 de marzo de 2020).

Knipper, Michael (2006). "El reto de la medicina intercultural y la historia de la medicina tradicional indígena contemporánea”, en Fernández Juárez, Gerardo (coord.). Salud e interculturalidad en América Latina. Antropología de la salud y crítica intercultural. Quito, Ecuador: Abya-Yala, pp. 413-432.

Kohler, Ulrich (1975). Cambio cultural dirigido en Los Altos de Chiapas. Un estudio sobre antropología social aplicada, Colección INI/Sep, Núm. 42. Chiapas, México: Instituto Nacional Indigenista, Secretaría de Educación Pública.

Lefebvre, Henry (1974). "La production de l'espace. L'Homme et la société". Sociologie de la connaissance marxisme et anthropolgie, Núm. 31-32, pp. 15-32. doi: https://doi. org/10.3406/homso.1974.1855 (Consultado el 4 de mayo de 2021).

Lomnitz Alder, Joaquín (1995). "Conceptos para el estudio de la cultura regional". Las salidas del laberinto. Cultura e ideología en el espacio nacional mexicano. México, D. F., México: Grupo Editorial Planeta, pp. 35-53

Lozoya, Xavier, Georgina Velázquez y Ángel Flores (1988). La Medicina Tradicional en México. Experiencia del Programa IMSS-COPLAMAR 1982-1987. México, D. F., México: Instituto Mexicano del Seguro Social.

Magaña Ochoa, Jorge (2002). "Enfermedad y tratamiento entre la población indígena migrada en la ciudad de San Cristóbal de Las Casas, Chiapas. Lo médico como campo social”. Relaciones Estudios de Historia y Sociedad, 23 (92), pp. 195-226.

Magaña Ochoa, Jorge (2016). "Medicina con Enfoque Intercultural: Entre el conflicto de prácticas discriminatorias y la toma de posición de un Modelo Médico por estudiantes Indígenas de Chiapas, México”, en Magaña, Jorge, et al. (coords.). Estudios Latinoamericanos: Pueblos originarios hacia el siglo XXI. Enfoques actuales. San Cristóbal de Las Casas, Chiapas, México: Universidad Autónoma de Chiapas, Consejo Latinoamericano de Ciencias Sociales, pp. 171-196.

EntreDiversidades. Revista de Ciencias Sociales y Humanidades, Vol. 9, Núm. 1 (18), enero-junio 2022. Páginas: 247-271 ISSN-e: 2007-7610. https://doi.org/10.31644/ED.V9.N1.2022.A10 
Rocío Marisela Ruiz Ruiz

José Enrique Eroza Solana

Jorge Magaña Ochoa
Configuración de la región sanitaria de la zona Altos Tsotsil-Tseltal: panorama...

Magaña Ochoa, Jorge y Rojas Trejo, Belkis (2020). “Algunas reflexiones desde la Antropología de la Salud en el contexto latinoamericano”. Boletín Antropológico [en línea], 38 (100), pp. 356-391. Disponible en: https://www.redalyc.org/journal/712/71266664005/ (Consultado el 18 de diciembre de 2021).

Menéndez, Eduardo L. (1992). "Modelo hegemónico, modelo alternativo subordinado, modelo de autoatención. Caracteres estructurales”. La antropología médica en México. México, D. F., México: Universidad Autónoma Metropolitana, pp. 97-113.

Menéndez, Eduardo L. (2016). "Salud intercultural: propuestas, acciones y fracasos". Ciência \& Saúde Coletiva [en línea], 21 (1), pp. 109-118. doi: https://doi.org/10.1590/141381232015211.20252015 (Consultado el 18 de diciembre de 2021).

Morales Domínguez, Magdalena (2021). "La experiencia materna de cinco mujeres de los Altos de Chiapas". La medicalización y la maternidad en los Altos de Chiapas. Chiapas, México: Universidad Autónoma de Chiapas, pp. 75-78.

Morales Domínguez, Magdalena (2021). "Reflexiones sobre la complejidad de la medicalización en la salud materna”. La medicalización y la maternidad en los Altos de Chiapas, Chiapas, México: Universidad Autónoma de Chiapas, pp.175-187.

Organización Mundial de la Salud (OMS) (2002). Estrategia de la OMS sobre Medicina Tradicional 2002-2005. Ginebra, Suiza: Organización Mundial de la Salud. Disponible en: http:// apps.who.int/iris/bitstream/handle/10665/67314/WHO EDM TRM 2002.1 spa. pdf;jsessionid=CE99684CFE774DF0EB50F1B215892812? sequence $=1$ (Consultado el 22 de julio de 2019).

Page Pliego, Jaime Tomás (2002). "Primera parte: Política sanitaria dirigida a los pueblos indígenas de México y Chiapas 1857-1995”. Política sanitaria dirigida a los pueblos indígenas de México y Chiapas 1857-1995. México, D. F., México: Programa de Investigaciones Multidisciplinarias sobre Mesoamérica y el Sureste, Universidad Nacional Autónoma de México, Instituto de Estudios Indígenas, Universidad Autónoma de Chiapas, pp. 21-66.

Ramírez Goicoechea, Eugenia (2011). Etnicidad, Identidad, Interculturalidad. Teorias, conceptos y procesos de la relacionalidad grupal humana. Madrid, España: Centro de estudios Ramón Areces, S. A., pp. 579-603.

Ruíz Ruíz, Rocío (2018). Interculturalidad en Salud: Parteras tradicionales y biomedicina. Casa materna y medicina tradicional en San Cristóbal de Las Casas, Chiapas. Tesis para obtener el grado de Maestría en Desarrollo Local. Facultad de Ciencias Sociales, Universidad Autónoma de Chiapas. También disponible en: http://www.repositorio.unach.mx:8080/ jspui/bitstream/123456789/3166/1/RIBC154346.pdf

Ruíz Ruíz, Rocío (2021). De encuentros ficticios a desencuentros reales: saberes y significados de pacientes sobre su padecimientolenfermedad en el sistema hospitalario del Hospital de Las Culturas. Borrador de tesis para obtener el grado de Doctora en Estudios Regionales. Universidad Autónoma de Chiapas.

Sánchez Ramírez, Georgina (2016). "Antecedentes". Espacios para parir diferente. Un acercamiento a Casas de parto en México. San Cristóbal de Las Casas, Chiapas, México: El Colegio de la Frontera Sur, pp. 15-30.

EntreDiversidades. Revista de Ciencias Sociales y Humanidades, Vol. 9, Núm. 1 (18), enero-junio 2022. Páginas: $247-271$ ISSN-e: 2007-7610. https://doi.org/10.31644/ED.V9.N1.2022.A10 
Rocío Marisela Ruiz Ruiz

José Enrique Eroza Solana

Jorge Magaña Ochoa
Configuración de la región sanitaria de la zona Altos Tsotsil-Tseltal: panorama...

Secretaría de Bienestar (2016). Guía de programas sociales 2016 [en línea]. Disponible en: https://www.gob.mx/bienestar/documentos/guia-de-programas-sociales-2016?idiom=es (Consultado el 28 de enero de 2020).

Servicio Internacional para la Paz (SIPAZ) (2014). Chiapas: El Colectivo de Mujeres (Colem) cumple 25 años [en línea]. Disponible en: https://sipaz.wordpress.com/2014/05/21/chiapas-elcolectivo-de-mujeres-colem-cumple-25-anos/ (Consultado el 14 de noviembre de 2021).

Secretaría de Salud (SSA) (2008). Servicios y unidades de Salud culturalmente competentes [en línea]. México: Gobierno de México. Disponible en: https://www.gob.mx/cms/uploads/ attachment/file/29316/ServiciosUnidades.pdf (Consultado el 10 de marzo de 2021).

Secretaría de Salud Chiapas (SSA) (2021). Unidades médicas. Chiapas [en línea]. Disponible en: https://saludchiapas.gob.mx/unidades-medicas (Consultado el 15 de noviembre de 2021).

Unidad 12 pasos (2019). ¿Qué son los grupos de $4^{\circ}$ y $5^{\circ}$ paso? [video]. Disponible en: https://www. youtube.com/watch? $v=3 \mathrm{mHGFbq0GLU}$ (Consultado el 12 de noviembre de 2021).

Viesca Treviño, Carlos (1987). "Veinte años de investigación en medicina tradicional en México", en Durán, S. (coord.). Medicina tradicional y atención primaria. Ensayos en homenaje a Gonzalo Aguirre Beltrán. México, D. F., México: Centro de Investigaciones y Estudios Superiores en Antropología Social: Cuadernos de la Casa Chata, pp. 59-70.

Villa Rojas, Alfonso (1976). "Introducción". El indigenismo en acción. México, D. F., México: Instituto Nacional Indigenista, pp.11-24.

Villa Rojas, Alfonso (1990). "Organización política y religiosa". Etnografía tzeltal de Chiapas. Modalidades de una cosmovisión prehispánica, México, D. F., México: Gobierno del Estado de Chiapas, pp. 23-40.

Volver a Vivir Libre, A.C. (2021). Historia [en línea]. Disponible en: https://volveravivirlibre. com/index.html (Consultado el 22 de noviembre de 2021).

EntreDiversidades. Revista de Ciencias Sociales y Humanidades, Vol. 9, Núm. 1 (18), enero-junio 2022. Páginas: $247-271$ ISSN-e: 2007-7610. https://doi.org/10.31644/ED.V9.N1.2022.A10 